\title{
Coulisses
}

Revue de théâtre

25| Hiver 2002

Varia

\section{Discours inaugural aux Arts de la scène}

Jean-Jacques Girardot

\section{(2) OpenEdition}

Journals

Édition électronique

URL : http://journals.openedition.org/coulisses/6041

DOI : 10.4000/coulisses.6041

ISSN : 2546-9460

\section{Éditeur}

Presses universitaires de Franche-Comté

\section{Édition imprimée}

Date de publication : 1 janvier 2002

Pagination : 105-106

ISBN : 2-84627-052-X

ISSN : $1150-594 \mathrm{X}$

\section{Référence électronique}

Jean-Jacques Girardot, « Discours inaugural aux Arts de la scène », Coulisses [En ligne], 25 | Hiver 2002, mis en ligne le 24 octobre 2019, consulté le 15 novembre 2019. URL : http://

journals.openedition.org/coulisses/6041 ; DOI : 10.4000/coulisses.6041

Ce document a été généré automatiquement le 15 novembre 2019.

Coulisses 


\title{
Discours inaugural aux Arts de la scène
}

\author{
Jean-Jacques Girardot
}

1 Je me réjouis beaucoup d'accueillir au nom de l'Université de Franche-Comté cette seconde journée « Les arts de la scène à l'Université ».

C'est un événement qui constitue la clé de voûte du développement, patient et tenace, de la filière artistique et culturelle, au sein du département des Sciences de l'Homme et de la Société de notre faculté des Lettres et Sciences Humaines.

2 Avec la maitrise Arts du spectacle-Études théâtrales, la filière théâtre offre depuis cette année un second cycle complet. Nous proposons également depuis la rentrée une licence de musique et une maîtrise d'archéologie. L'an dernier, parallèlement à la licence Arts du spectacle-Études théâtrales, nous avions ouvert, outre la licence d'archéologie, la licence professionnelle en Développement et protection du patrimoine culturel «Métiers de l'exposition et technologies de l'information " (METI). Le deug Arts du spectacle et le deust Arts du spectacle-théâtre avaient été créés lors du précédent quadriennal, en 1996, pour former l'embryon de la section Arts avec le deug de musique habilité depuis 1984, l'année où nous avions rénové les deug. Pour demain, un DEA en Théâtre et une jeune équipe en Arts sont les projets d'avenir inscrits au contrat de plan dans le cadre de la Maison des Sciences de l'Homme. Je pense au chemin parcouru quand j'aperçois aujourd'hui dans la salle de très nombreux acteurs de cette aventure. Je me souviens de cette rencontre avec Jacques Vingler, directeur du Centre de rencontres de Besançon, Bernard Carraud du Conseil régional de FrancheComté et Lucile Garbagnati dans le petit bureau de littérature comparée. J'étais alors assesseur aux formations. Cette rencontre avait pour but de créer un diplôme d'université en métiers du spectacle sur le modèle du diplôme d'université Assistants Artistiques, dont j'avais établi le dossier à l'initiative de François Cheval, alors directeur du Fonds régional d'art contemporain de Franche-Comté, avec le soutien actif de Bernard Carraud et de l'École régionale des Beaux-Arts.

3 Trois diplômes d'université avaient finalement été habilités en 1989 : le DU Pédagogie de la musique, le DU Assistants artistiques et le DU Métiers du spectacle. Le DUPM se 
poursuit actuellement à Dijon. Le DUAA est devenu la licence professionnelle METI l'an passé. L'habilitation du DUMST comme DEUST, et celle du DEUG Métiers du spectacle ont posé les bases de la filière théâtre.

4 Ces diplômes, tout particulièrement ceux qui composent la filière théâtre, sont le fruit d'un partenariat actif avec les institutions locales publiques et territoriales et avec les structures professionnelles.

5 La DRAC, le Conseil régional et la ville de Besançon, nous ont soutenus financièrement jusqu'à ce que les diplômes d'université soient habilités par le ministère et reçoivent une dotation de l'État. Je souligne que le soutien financier des partenaires locaux, qui ne s'est jamais démenti pendant douze années, se poursuit pour élever le niveau de qualité de nos formations. Je veux remercier ici les présidents, les directeurs, les attachés et les chargés de mission qui ont défendu les métiers de l'art. Ils ont aussi illustré l'importance de la culture dans le développement territorial.

6 L'implication de partenaires culturels et professionnels est également remarquable. Le Fonds régional d'art contemporain, l'École des Beaux-Arts, le Conservatoire de musique et, plus particulièrement pour le théâtre, le Centre de rencontre, puis l'Embarcadère, L'Espace et, à présent, le Nouveau Théâtre, CDN de Franche-Comté, ont toujours été présents en vertu d'un principe simple : les étudiants doivent être «sur le pont ». Tous ces organismes ont été directement impliqués dans la définition des programmes et dans l'organisation matérielle des enseignements, notamment des enseignements professionnels. Ils ont accueilli les étudiants qui ont pu profiter de leurs équipements et de leurs compétences. Notons que la synergie de ces partenaires intervenant dans des disciplines artistiques complémentaires a favorisé l'émergence de projets originaux.

7 On peut difficilement imaginer le nombre de personnes que je souhaite remercier maintenant et la diversité de leurs métiers. Je pense également à toutes les structures, les associations et les professionnels qui ont enrichi nos enseignements et qui ont accueilli des stagiaires.

Nous devons aussi remercier tous ceux qui ont défendu ces formations au sein de l'Université : Jean-Philippe Massonie, auquel j'ai succédé à la direction de l'UFR, Claude Condé, le doyen actuel, et François Favory, directeur du département des Sciences de l'Homme et de la Société. Nous voulons aussi associer tous ceux, enseignants et personnels administratifs, qui ont fait fonctionner quotidiennement ces enseignements depuis douze ans. Une mention particulière pour Françoise Quillet, responsable de la filière théâtre, et Olivier Thévenin, responsable du DEUST Métiers du spectacle (et de la LP METI). Ils se sont très fortement impliqués, avec une énergie débordante, dans les diplômes dans lesquels nous les avons plongés dès leur arrivée à l'université.

Je ne remercie pas toutes ces personnes par simple convenance, mais par amitié, avec complicité. Je n'ai pas pu toutes les citer. J'ai certainement omis de mettre en valeur le nom de nombreux soutiens déterminants. Je souhaite évoquer le nombre et la diversité de ceux qui ont soutenu le développement de la filière culturelle et plus particulièrement des formations en théâtre. Tous ces acteurs ne sont pas des gens de théâtre. Pour ma part, économiste de formation, actuellement responsable d'une formation en informatique et technologies de l'information, j'ai pris ma part de cette aventure, celle de l'administration : montage des dossiers, calcul des budgets, rédaction 
des argumentaires. Aussi ai-je en ce moment une reconnaissance plus particulière pour tous ceux qui ont œuvré dans les coulisses de l'assistance technique et administrative.

Le fait que le Nouveau Théâtre, partenaire très actif, accueille aujourd'hui cette journée de réflexion me donne l'occasion de monter sur la scène. Que Michel Dubois, son directeur, et Guillaume Dujardin, assistant à la mise en scène et professeur associé soient remerciés.

11 En voyant les participants, nombreux et attentifs, qui occupent aujourd'hui les sièges des spectateurs, je ressens bien ce capital de solidarité et de sympathie qui a fortement contribué au succès de nos entreprises dans le domaine théâtral. Nous sommes bien ici dans le lieu propice à une réflexion sur les objectifs des formations et des cursus des Arts de la scène.

\section{AUTEUR}

\section{JEAN-JACQUES GIRARDOT}

Vice-Président de l'Université de Franche-Comté 